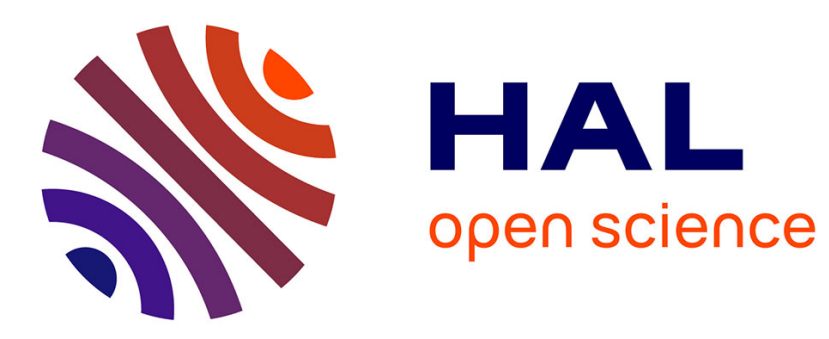

\title{
Samuel Gaudon, sieur de la Rallière, un financier protestant au service de Mazarin
}

Guillaume Métayer

\section{To cite this version:}

Guillaume Métayer. Samuel Gaudon, sieur de la Rallière, un financier protestant au service de Mazarin. Revue d'histoire du protestantisme, 2021, 6 (3), pp.289-310. hal-03435752

\section{HAL Id: hal-03435752 \\ https://hal.science/hal-03435752}

Submitted on 18 Nov 2021

HAL is a multi-disciplinary open access archive for the deposit and dissemination of scientific research documents, whether they are published or not. The documents may come from teaching and research institutions in France or abroad, or from public or private research centers.
L'archive ouverte pluridisciplinaire HAL, est destinée au dépôt et à la diffusion de documents scientifiques de niveau recherche, publiés ou non, émanant des établissements d'enseignement et de recherche français ou étrangers, des laboratoires publics ou privés. 


\title{
Samuel Gaudon, sieur de la Rallière, un financier protestant au service de Mazarin
}

\author{
Guillaume MÉTAYER
}

Personnalité médiatique mais oubliée du XVII ${ }^{\mathrm{e}}$ siècle, Samuel Gaudon (1594-1650) a pu bénéficier d'un nouvel éclairage à travers l'étude de son château dans le cadre d'un mémoire de master ${ }^{1}$. Pendant longtemps la perception qu'on en avait fut biaisée car elle se limitait à la compilation des mazarinades le mentionnant. Ces pamphlets, largement diffusés pendant la Fronde (1648-1653) et souvent violents à l'égard de Mazarin et des financiers, mentionnent un « La Raillière » qui n'est pas présenté sous son meilleur profil. Le renouvellement historiographique ne débute qu'à partir de la fin des années 1970, lorsque Daniel Dessert ${ }^{2}$ et Françoise Bayard ${ }^{3}$ s'intéressent à un champ peu ou pas étudié, le monde des financiers du XVII ${ }^{\mathrm{e}}$ siècle. Les mazarinades sont laissées de côté au profit des sources archivistiques, ouvrant la voie à une meilleure compréhension du système financier de l'époque ${ }^{4}$. En effet, la vie de Samuel Gaudon est éclairée par les centaines d'actes notariés dans lesquels il apparaît. Ces actes attestent de son aisance financière et de sa capacité à traiter autant avec des bourgeois protestants qu'avec la noblesse parlementaire, mais ils laissent de côté une grande partie de sa vie personnelle. Le recours aux sources notariales permet de nuancer les mazarinades et d'étudier sa vie sur une période plus large, tout en le resituant au cœur du milieu financier protestant des années 1630-1640. La Fronde, qui d'après l'historiographie l'avait ruiné et avait brisé sa carrière (ce qui devait expliquer l'inachèvement de son château à Preuilly), le met en fait sur le devant de la scène, où il brille par son éloquence dans la défense des intérêts des financiers. Sa mort brutale intervient alors qu'il est en pleine ascension, laissant ouvertes toutes les hypothèses quant à ce qu'il aurait pu devenir quelques années plus tard.

\section{De Preuilly à Paris via Rochefort}

Fils d'un lieutenant ordinaire de la baronnie de Preuilly, deuxième pôle protestant de Touraine, Samuel Gaudon naît le 23 avril et est baptisé le 12 juin 15945. Un an auparavant, l'humaniste Joseph Juste Scaliger quittait le château de Preuilly dans lequel il était hébergé par les Chasteigner depuis 1590. Il avait notamment contribué à la conversion des principaux notables de la baronnie (dont les parents de Samuel Gaudon en 1593). Les sources concernant la jeunesse du futur financier sont rares, et se pose notamment la question de sa formation : si le fonds de l'Académie protestante de Saumur ne conserve aucune trace de son passage ${ }^{6}$, c'est peut-être que son père François Gaudon préféra lui faire suivre un enseignement plus traditionnel, tel que celui dont luimême avait pu bénéficier. En effet, comme lui, Samuel est diplômé de l'Université de Poitiers, où il

1 Guillaume MÉTAYER, Le château de la Rallière, Mémoire de master en histoire de l'art sous la direction d'Alexandre Gady, Sorbonne Université, 2019.

2 Daniel DESSERT, « Le « laquais-financier » au Grand Siècle : mythe ou réalité ? », Dix-septième siècle 122 (1979), p. 21-36.

3 Françoise BAYARD, Le monde des financiers au XVII siècle, Paris : Flammarion, 1988, $621 \mathrm{p}$.

4 Françoise BAYARD, "L'image littéraire du financier dans la première moitié du XVII ${ }^{\mathrm{e}}$ siècle », Revue d'histoire moderne et contemporaine 33 (1986), p. 3-20.

5 AD Indre-et-Loire, Preuilly, Église réformée (1590-1679).

6 AM Saumur : http://archives.ville-saumur.fr/f/acaprotestante/mosaique/?\&reset criteres=1 
obtient les 17 et 18 novembre 1615 le grade de bachelier et licencié «in utroque jure ${ }^{7}$. L'inventaire après décès de son épouse en $1643^{8}$ nous offre un aperçu de sa bibliothèque. Les ouvrages de géographie, d'art militaire, d'histoire, de droit, ainsi que les bibles et traités théologiques, témoignent de la formation sérieuse de Samuel Gaudon, rompant avec l'image du « laquais financier » inculte et imbécile qui englobe généralement tous les financiers du XVII ${ }^{\mathrm{e}}$ siècle, et dont Françoise Bayard et Daniel Dessert ont pu montrer les incohérences. En outre, son appétence pour la littérature savante se retrouve dans la dédicace que lui fait son cousin Sylvain Gaudon ${ }^{9}$, qu'il héberge quelque temps dans son hôtel parisien :

Je vous supplie tres-humblement de donner quelque heure des nuicts que vous passez à la lecture de toute sorte de bons Livres, pour vous delasser des grandes affaires qui vous occupent tous les jours; de donner, dis-je, un peu de temps à parcourir les Ambassades et Voyages de Busbequius que je vous offre: où je ne doute point que vous ne receviez beaucoup de satisfaction, par la cognoissance très exacte que vous avez, et Politique et Geographique, de tous les Estats de la terre : des Histoires Anciennes et Modernes : et de tous les interests des Princes.

Le début de sa carrière est certainement contemporain de son achat du fief de la Rallière, dont il conserve le titre jusqu'à sa mort, bien qu'il ait revendu la propriété en $1634^{10}$. En effet, dans un acte de baptême du 17 juillet 1616, il n'est présenté que sous son patronyme, mais le 4 octobre 1622, pour le baptême de Pierre, fils de son cousin Abel Piozet et d'Adriane Lefort, il est qualifié de « sieur de la Ralière"1" ". C'est donc avant 1622 qu'il achète « la terre et seigneurie de la Raillière [...] en la paroisse de Precigny pays de Touraine ${ }^{12} »$, se servant peut-être de l'héritage de son père mort dix ans plus tôt, ce qui lui donne, d'une certaine manière, un « nom de scène » que les Parisiens retiendront plus que les Prulliaciens.

Il n'a pas été possible de retracer le parcours de Samuel Gaudon dans les années qui suivent l'obtention de son diplôme. Il ne réapparaît que huit ans plus tard, le 20 juin 1623, comme contrôleur triennal au grenier à sel de Château-Gontier ${ }^{13}$. C'est seulement à partir de 1627 que les actes le mentionnant se multiplient, ce qui peut s'expliquer par sa présence à Paris, où les archives notariales sont mieux conservées pour cette période. Dans son premier cercle de relations se trouvent principalement des seigneurs du sud de la Touraine et de l'ouest du Berry, protestants, dans l'orbite de Louis d'Aloigny, marquis de Rochefort (à $30 \mathrm{~km}$ au sud de Preuilly). Le 4 novembre 1627, il prête 800 livres à Gabriel de Gébert, seigneur de Noyant de Touraine. Le 30 janvier 1628, devant notaire, ce dernier promet de le rembourser. À cette occasion, il est logé « en la maison de monsieur le marquis de Rochefort ${ }^{14} »$, ce qui témoigne du rôle du marquis dans les premières affaires financières de Samuel Gaudon. Le même jour, il traite avec Abel et Pierre de Sainte-Marthe

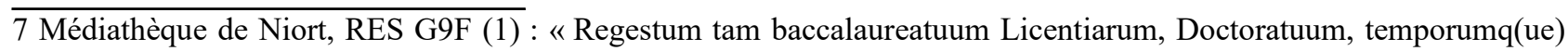
studiorum, quam aliorum actuum expeditorum in Collegio facultatis Canonici et Ciuilis jurium huius almae ac fructiferae Universitatis Pictavensis ». Registre manuscrit de l’Université de Poitiers entre 1605 et 1615 , f221 r, $\mathrm{n}^{\circ} 1308$.

8 AN, Minutier central, Étude XXI, 205.

9 AD Indre, 48J 5 C.

10 À Jacques Papelard, barbier chirurgien bourgeois de Paris, dans le cadre d'une transaction qu'il effectue en association avec plusieurs grands officiers du Mans dans le but d'acquérir les offices de « procureur du roy alternatif, second advocat du roy et commissaires examinateurs des eslections et generalité de Touraine » (AN, Minutier central, Étude XXI, 124).

11 AD Indre-et-Loire, Preuilly, Église réformée (1590-1679).

12 AN, Minutier central, Étude XXI, 124.

13 AN, $Z^{1 \mathrm{~A}} 148$, Répertoire de la cour des Aides.

14 AN, Minutier central, Étude LI, 152. 
et Henri d'Harambure ${ }^{15}$. Le frère de ce dernier épouse Marie Tallemant, cousine de Gédéon Tallemant des Réaux, l'année suivante. Le château principal des Harambure est celui de Romefort, proche des châteaux de Rochefort et de Preuilly. Le $1^{\text {er }}$ février 1630, Samuel Gaudon est clairement présenté comme « demeurant à Paris rue Beaubourg paroisse St Merry en la maison de monsieur le marquis de Rochefort ${ }^{16} \gg$. Ce dernier l'a peut-être repéré à Preuilly, l'a initié aux pratiques financières (étant lui-même très introduit dans le milieu de la finance) avant d'en faire son « intendant des maison et affaires ${ }^{17}$ ». Il est donc logique de le retrouver régulièrement à Craon, en Mayenne, dont le marquis de Rochefort était le seigneur.

L'ascension de Samuel Gaudon ne pouvait reposer sur le seul marquis de Rochefort. Peutêtre lui conseilla-t-il le mariage, impossible avant qu'il eût atteint un certain statut. Son contrat de mariage avec Anne Menjot est signé le 7 mars 1630, dans la maison du marquis de Rochefort ${ }^{18}$. Le 19 janvier, sa future belle-mère, Anne Mallard, lui prête 8000 livres $^{19}$, peut-être en lien avec les 10 000 livres qu'il devait apporter à la communauté dans son contrat de mariage. Le marquis de Rochefort promet la même somme, qu'il ne paiera que le 20 juin 1645. L'épouse, quant à elle, apporte 27000 livres, divisées en 10000 pour la communauté et 17000 qu'elle conserve pour son usage propre, ainsi que 4500 livres « en bagues, vaisselle d'argent et autres meubles ». Les Menjot étaient de noblesse de robe. À son mariage Anne était veuve de Christophe Petit, avocat en parlement, proche du prince de Condé. Son statut de veuve a peut-être favorisé ce second mariage qui semble moins important que le premier, mais il est fort probable que chacun des conjoints y ait trouvé son compte. Anne Menjot n'apporte pas une dot considérable, mais elle est affiliée aux Monceau (dont Isaac, qui est présenté par Tallemant des Réaux comme étant « un des plus riches garçons de Charenton », et avec lequel Samuel Gaudon va régulièrement collaborer), aux Rambouillet et aux Tallemant, qui dominent la finance protestante.

Ce mariage marque une nouvelle étape dans sa carrière. La prise d'autonomie du couple apparaît dans un bail de location du 9 septembre 1630 d'un hôtel entre cour et jardin rue des Ménétriers, pour 450 livres par $a^{20}$. Le 18 juin 1631, les syndics généraux des gabelles de France organisent leur assemblée « en la maisons dud. sieur de la Rallière par la convocation des sieurs Phellippes et Cappon syndics généraux pour délibérer sur les affaires concernant leur communauté ${ }^{1}$ ». Samuel Gaudon est présenté comme « receveur des gabelles au grenier à sel de Craon, tant en son nom que comme ayant charge et se tenant fort des sieurs receveurs particuliers des gabelles de la généralité de Touraine ». Le marquis de Rochefort reste donc présent en arrièreplan via sa baronnie de Craon. En réunissant l'assemblée chez lui, et en représentant les receveurs particuliers des gabelles de la généralité de Touraine, Samuel Gaudon montre son influence et sa prise d'initiative qui le font se démarquer.

\section{Le bail général des aides de France}

En 1634 Samuel Gaudon devient Fermier général des aides de France et abandonne (officiellement) le marquis de Rochefort (sa dernière mention en tant qu'intendant date du 22 avril

\footnotetext{
15 Ibid.

16 AN, Minutier central, Étude LI, 158.

17 AN, Minutier central, Étude LI, 164.

18 AN, Minutier central, Étude LI, 158.

19 Ibid.

20 AN, Minutier central, Étude LI, 160.

21 AN, Minutier central, Étude LI, 162.
} 
1634 $)^{22}$. Le bail général de toutes les aides de France, entreprise monumentale de perception d'impôts préfigurant la Ferme générale créée par Louis XIV en 1680, suppose un solide socle de bailleurs de fonds. Elle est affermée pour neuf ans, à compter du $1^{\text {er }}$ avril 1634, à François Chandonnay, qui fait office de prête-nom pour une société réunissant Étienne Briois, sieur de Bagnolet, André Legrand, Jean Philippes, Jean Favarel et Samuel Gaudon ${ }^{23}$. La gestion de la ferme nécessite une organisation bien réglée. Un traité est passé le 13 avril entre Jacques Bordier et Étienne Briois pour les offices de contrôleurs conservateurs des Fermes de France ${ }^{24}$. Ces offices pouvaient être acquis, et donnaient la qualité d'officier contrôleur conservateur, chargé de veiller à la bonne perception de l'impôt et rémunéré par les fermiers des aides. Le traité est confirmé le 22 mai par les autres associés à la Ferme. Par un traité du 20 novembre, Samuel Gaudon et Jean Philippes s'octroient les offices de contrôleurs conservateurs des aides de la généralité de Tours (Jean Philippes lui cède sa moitié le 2 septembre 1638) ${ }^{25}$. De nombreux baux se succèdent ainsi jusqu'à la fin du bail général. Malgré les rentrées d'argent censées être régulières, les fermiers doivent payer leur bail et rémunérer leurs officiers, ce qui mobilise d'importantes sommes. Le 8 septembre 1634 Jean Habert, sieur du Mesnil, leur prête 252000 livres, qui lui sont remboursées un an plus tard ${ }^{26}$. Il s'agit du père de Marie Habert, épouse de Louis d'Aloigny, marquis de Rochefort, ce qui a pu faciliter le prêt. Le 7 septembre 1635 Jean de Choisy, sieur de Balleroy, leur prête 102 000 livres $^{27}$. Le 29 juillet 1643, lors de la rédaction de l'inventaire après décès d'Anne Menjot, Samuel Gaudon déclare que le bail de la ferme est terminé depuis le 31 novembre 1634, que « le compte n'a pas encore esté rendu par $\mathrm{M}^{\mathrm{e}}$ Sulpice Cuper commis a la Recepte Generalle de ladite Ferme » et qu'il « ne peult dire environ la perte qu'il y peult avoir sinon bien assurement qu'il [rayé : y a plus d'ung million de perte] s'en fault plus d'un million qu'il n'y ayt de proffict $^{28} »$. Le 24 août 1646 Isaac Monceau fournit 216815 livres «pour supléer au manque de fondz qui s'est trouvé en ladite ferme ${ }^{29}$ ». Peut-être Samuel Gaudon eut-il aussi à fournir les mêmes sommes. Il est nécessaire de participer à plusieurs baux afin de compenser ceux qui s'avèrent coûter plus qu'ils ne rapportent.

La gestion de la Ferme n'empêche pas Samuel Gaudon de participer à d'autres traités. Le 21 juin 1634, il reçoit les lettres de provision signées du roi pour l'office de conseiller examinateur en l'élection d'Angers, acquis 4042 livres conjointement avec son épouse Anne Menjot ${ }^{30}$. De 1636 à 1639, il est procureur des biens de François de Vignerot de Pont-Courlay, général des Galères et neveu maternel de Richelieu ${ }^{31}$. En décembre 1636, il est chargé de recevoir 24000 livres sur les 42 000 auxquelles François de Cossé duc de Brissac, qui «y sera contraint par toutes voyes dues et raisonnables mesme par emprisonnement de sa personne », a été condamné ${ }^{32}$. Le 16 janvier 1639, «à sa priere et pour luy faire plaisir », Samuel Gaudon promet 10090 livres à Picart, trésorier général de la Marine du Levant ${ }^{33}$.

Cet accroissement de son activité, parallèlement à la naissance de ses enfants (Auguste en 1632, Madeleine en 1633, Paul en 1636 et Anne en 1639), nécessite un agrandissement de son hôtel 22 AN, Minutier central, Étude XXI, 124.

23 AN, Minutier central, Étude XXI, 209.

24 Ibid.

25 Ibid.

26 AN, Minutier central, Étude LI, 105.

27 AN, Minutier central, Étude LI, 107.

28 AN, Minutier central, Étude XXI, 205.

29 AN, Minutier central, Étude XXI, 209.

30 AN, Minutier central, Étude XXI, 205.

31 Ibid.

32 AN, Minutier central, Étude XXI, 129.

33 AN, Minutier central, Étude XXI, 205. 
(en 1635 34 ). Le 27 janvier 1637, il loue à Claude Bouteroue une nouvelle maison, toujours rue des Ménétriers, « consistant en deux corps d'hostel, l'une sur le devant l'autre sur le derrière cours au milieu escurye en icelle » pour 6 ans et 500 livres par an ${ }^{35}$. Ainsi, même s'il change d'hôtel, il reste dans la même rue, proche de l'hôtel de Rochefort.

Contrebalançant cette gestion financière irréprochable, un événement a contribué à bouleverser l'image de Samuel Gaudon, faisant de lui un personnage fantasque et extravagant : le 5 septembre 1638, à l'annonce de la naissance du Dauphin, futur Louis XIV,

Le sieur La Ralière fit ouvrir chez lui une fontaine à quatre canaux, chacun d'un pouce de diamètre, y fit couler, depuis midi jusqu'à deux heures après minuit, vingt-six muids de vin exquis, ayant deux longues tables dans la rue, au dessous des canaux, chargées de jambons, cervelas, pastez, gorge de porc et autres semblables aiguillons à boire, où l'on ne s'épargnoit pas. Et toutefois, non content de cela, il se promena une grande partie de la nuit avec deux carrosses pleins de violons, hauts-bois et musiciens, suivis d'un chariot chargé de trois muids de vin en bouteilles et de pareilles viandes et pasticeries, dont il faisoit present à tous les passans, et en laissa chez plusieurs de ses amis. Bref, il faut avoir vu cette liesse pour la croire ${ }^{36}$.

La précision des détails rend difficile l'hypothèse d'une fiction imaginée par le gazetier, mais cette fête et ce débordement sont à l'opposé de la rigueur dont rendent compte les minutes notariales. Il faudrait donc y voir une mise en scène bien calculée de Samuel Gaudon, cherchant à manipuler les Parisiens en faveur du roi, peut-être dans l'espoir que son action arrive jusqu'aux oreilles de Richelieu ou du souverain. Il renouvelle l'expérience pendant la Fronde, sous les ordres de Mazarin, en distribuant du vin et de l'argent « aux bateliers et à d'autres gens de la lie du peuple ${ }^{37}$ » afin de préparer l'entrée royale et cardinale dans la ville.

\section{Taxe des Aisés et retour à Preuilly}

La mort d'Anne Gaudon le 14 avril $1643^{38}$, suivie un mois plus tard par celle de Louis XIII, inaugure une période capitale de la vie de Samuel Gaudon. Désormais veuf, il s'occupe de ses enfants tout en multipliant les traités et en renouvelant sa participation à la Ferme générale des aides de France. Son aisance financière étant assurée, il va diversifier ses activités. Cette assurance se perçoit notamment dans un témoignage d'Olivier Lefèvre d'Ormesson rapportant les débats parlementaires sur la création d'une taxe d'Aisés en septembre $1644^{39}$, faisant suite à l'édit du Toisé de janvier 1644 qui avait déjà provoqué une révolte des parlementaires ${ }^{40}$. Mazarin souhaitant faire payer les plus riches parisiens, les parlementaires se défendent et veulent faire payer uniquement les financiers. Le samedi 3 septembre, Samuel Gaudon, "à la teste de tous les gens d'affaires ", parle « fort hardiment» à la reine. Il lui annonce « que si on les abandonnoit, ils ne payeroient plus les

34 AN, Minutier central, Étude XXI, 126.

35 AN, Minutier central, Étude XXI, 130.

36 Gazette de France, septembre 1638, n 127, p. 528, citée par Charles-Louis LiVET, Euvres complètes de SaintAmant, t. 2, nouvelle édition précédée d'une notice accompagnée de notes, Paris : P. Jannet, 1855, p. 498.

37 Gabriel DANIEL, Histoire de France, depuis l'établissement de la monarchie françoise dans les Gaules, nouvelle édition, revue, corrigée, et considérablement augmentée, t. XXII, Amsterdam et Leipzig : Arkstee \& Merkus, 1758, p. 20-21.

38 AN, Minutier central, Étude XXI, 205.

39 Olivier LEFÈVRE D’ORMESSON, Journal, publié par Adolphe Chéruel, t. I, Paris : Imprimerie impériale, 1860, p. $214-$ 215.

40 Michel Pernot, La Fronde, 1648-1653, Paris : Éditions de Fallois, 1994, p. 51-52. Ouvrage réédité à Paris : Tallandier, 2019. 
rentes de la ville, qu'ils ne feroient plus d'affaires et que l'on devoit considérer que messieurs du parlement ne pouvoient mieux faire pour ruiner ses affaires ; qu'ils en vouloient à son autorité, et que l'on distinguoit les parlementaires d'avec les royalistes; que l'exemple d'Angleterre devoit faire songer à elle. Il ajouta que la reyne avoit promis de les soutenir ». La gazette précise que «la Raslière, a bien osé, en parlant à M. le cardinal Mazarin, faire comparaison du parlement à celui d'Angleterre ; ce que S. Em. n'approuvant pas, dit qu'il y avoit grande différence, l'un estant pour conserver l'autorité du roy et l'autre contre ${ }^{41} »$. L'intervention de Samuel Gaudon a l'effet escompté («Les partisans ont fait grand bruit; leurs remonstrances ont esté escoutées ${ }^{42} »$ ) et permet à Mazarin et à la reine de montrer leur soutien aux financiers.

Une fois la taxe approuvée, sa mise en place ne va pas sans difficulté. Les aisés parviennent à négocier lorsqu'ils connaissent les bonnes personnes. Ainsi Amelot de Chaillou, conseiller au Grand Conseil, prie Lefèvre d'Ormesson « de voir M. de la Rallière, pour accommoder l'affaire de Mme Jappin $^{43}$ », taxée aux aisés à 1800 livres, et chez qui les traitants avaient envoyé une garnison, reçue par le cocher « qui, avec tous les valets du quartier, chassa et battit bien la garnison. Pour raison de quoy l'on procédoit criminellement contre eux ». Le lendemain, vendredi 4 août 1645, Ormesson s'entretient avec Samuel Gaudon, qui lui promet d'accommoder l'affaire «pourvu que dans le public l'autorité du roy fust rétablie ». Il conseille à ce propos de «prendre un domestique prisonnier, que l'on feroit sortir deux jours après ». Le témoignage de Jean Vallier confirme l'usage de la force pour contraindre au paiement des personnalités acceptant difficilement de devoir participer au renflouement des caisses de l'État « sur la foi et sur le rapport seulement de certains partisans (parmi lesquels le nommé Samuel Godon, sieur de la Rallière, paroissoit le plus insolent et dangereux $)^{44} »$.

À Paris, il lui faut une demeure encore plus grande que les précédentes. Le 27 janvier 1645, il loue à Adriane Lhermite « une grande maison [...] consistant en plusieurs corps de logis, cours devant et derrière, grande porte cochère sur ladicte rue de Beaubourg et qui a une autre sortie sur lad. rue des Petits Champs » pour 3 ans et 2000 livres de loyer par an ${ }^{45}$. C'est dans cette maison qu'est signé le contrat de mariage entre sa fille Madeleine (13 ans) et Georges de Clermont, comte de Saint-Aignan (23 ans) le 29 juin $1646^{46}$. Par ce mariage prestigieux, Samuel Gaudon introduit sa famille dans une classe sociale supérieure, qui atteste de sa réussite. Le marquis de Clermont, père du marié, leur offre le château du marquisat de Gallerande et de nombreuses terres et seigneuries en Anjou, Maine et Champagne. Samuel Gaudon, de son côté, offre une dot de 120000 livres, consistant en sa part d'héritage (ce qui permettra de mettre sa fille à l'abri des créanciers à sa mort), et qu'il promet de mettre en mains de son beau-père pour « l'employer au payement de ses debtes ». Le bien-être de sa fille reste au cœur de ses préoccupations et, le 29 mai 1648, il lui fait don de 90 000 livres, en plus de sa dot, « pour l'amour paternel qu'il luy porte, et par ce qu'ainsy il luy a pleu et luy plaist », à la condition que cette somme lui demeurera propre ${ }^{47}$. Il pressent que les difficultés financières des Clermont sont insolubles et susciteront de nombreux procès. Le même jour il est reçu conseiller secrétaire du roi ${ }^{48}$, événement qu'il souhaite donc commémorer par cette généreuse donation.

41 Gazette semi-officielle citée par Adolphe Chéruel en note de : Olivier LEFÈVRE D’ORMESSON, Journal, p. 214.

42 Ibid.

43 Ibid., p. 301-302.

44 Henri Courteault, Journal de Jean Vallier, maître d'hôtel du roi (1648-1657), t. I, Paris : Renouard, 1902 , p. 2-7. 45 AN, Minutier central, Étude XXI, 147.

46 AD Sarthe, 4 J 34.

47 AN, Minutier central, Étude XXI, 153.

48 Abraham TESSEREAU, Histoire chronologique de la Grande Chancellerie de France, Paris : Pierre Emery, t. I, 1710, p. 460 . 
Cette période est également marquée par son retour à Preuilly, qu'il ne semble pas avoir beaucoup fréquenté avant son veuvage. Le 31 mars 1644, il achète une maison vendue par sa sœur Marguerite et son beau-frère Josias Poizay pour 2400 livres. Sa présence à Preuilly est également attestée dans les registres protestants, sur lesquels il apparaît comme parrain le 19 août 1644, tout comme ensuite ses enfants Madeleine et Paul jusqu'au 17 avril $1650^{49}$. Cet achat en annonce d'autres, qui datent le début du chantier du château de la Rallière. L'étude de l'inventaire après décès de Samuel Gaudon permet de trouver la mention de plusieurs archives concernant ce chantier, les plus parlantes étant les « estat[s] de compte de recepte et despenses faictes par ledit Poizay pour ledit deffunt sieur de la Rallière en ses bastiments ${ }^{50} »$. Ces mentions attestent de la gestion du chantier depuis Preuilly par sa sœur Marguerite. Le premier «estat», clos le 8 février 1645, concerne l'année 1644. Après la mort de Josias, c'est son fils qui gère le chantier « à la requeste de Marguerite Gaudon sa mère ». Le dernier état est clos le 9 novembre 1648. L'étude des registres paroissiaux permet de repérer de nombreux ouvriers sur le territoire de la baronnie ces mêmes années. De plus, une étude dendrochronologique ${ }^{51}$ a montré que les arbres utilisés pour la charpente du pavillon sud ont été coupés, pour les derniers, durant l'automne-hiver 1646-1647, ce qui permet d'envisager une mise en place dans le courant de l'année $1647^{52}$. Le 20 novembre 1647, entendant « recognoistre les grandes et officieuses assistances que luy et les siens ont cy devant receues », il offre à sa sœur Marguerite, veuve depuis un mois, la maison de la Valette (qu'il lui avait acheté trois ans plus tôt) et, à sa nièce Marie Poizay (11 ans), 10000 livres qu'elle recevra lors de son mariage ${ }^{53}$. Ainsi le chantier de la Rallière atteste de moyens financiers importants et de la volonté de se rapprocher de sa baronnie natale. Pourtant, le programme du château, complété par un projet urbanistique matérialisé par une rue de sept pavillons identiques, sera revu à la baisse, et les occupations de Samuel Gaudon durant les deux dernières années de sa vie vont être trop prenantes pour lui permettre de quitter Paris.

\section{Du Parlement à la Bastille}

La réception de Samuel Gaudon comme conseiller secrétaire du roi intervient durant les prémices de la révolte parlementaire. Ainsi, le 18 juillet 1648 le Parlement révoque les intendants et Mazarin est contraint d'abaisser de 15 à $6 \%$ l'intérêt versé par l'État à ses créanciers et de reculer la date des remboursements ${ }^{54}$. Un débat oppose ceux qui voudraient repousser de vingt ans le remboursement des prêts des financiers au roi (pour lequel il faudrait utiliser tous les revenus ordinaires et extraordinaires des années 1648 à 1650) ${ }^{55}$ à ceux qui cherchent d'autres moyens de les indemniser. Les partisans de cette dernière option sont généralement liés à des financiers par des mariages pécuniairement intéressants, ou bien se sont associés à eux pour les prêts. Cette agitation dépasse le cadre parlementaire. Samuel Gaudon s'y invite par le biais d'un factum qu'il fait imprimer et diffuser, dans lequel il « débita un extrait de Cicéron, pro lege Manilia, où il montre 49 AD Indre-et-Loire, Preuilly, Église réformée (1590-1679).

50 AN, Minutier central, Étude XXI, 209.

51 Étude réalisée en 2018 par M. Benoît Brossier, Ingénieur d'études CNRS, Université de Montpellier (non publiée). 52 Le bois semble mis en œuvre tout de suite après son abattage, comme en témoigne la charpente du palais abbatial de Fontevraud, dont le devis est signé en juillet 1648, les bois datés par dendrochronologie de 1648-1649, et le toisé daté du 19 janvier 1649 : Jean-Yves HUNOT, «La chaîne opératoire, approche archéologique. De la forêt à la charpente : le savoir-faire du charpentier en Anjou », dans Patrick HOFFSUMMER (dir.), Les charpentes du XI au XIXe siècle, Grand ouest de la France, Turnhout : Brepols, 2011, p. 42.

53 AN, Minutier central, Étude XXI, 152.

54 M. PERNOT, La Fronde, 1648-1653, op. cit., p. 78.

55 Pierre ClÉMENT, Lettres, instructions et mémoires de Colbert, t. II, $1^{\text {re }}$ partie, Paris : Imprimerie impériale, 1863, p. 23. 
l'utilité et nécessité des publicains en une République, et un autre de Tacite, à la fin du XIII livre des Annales, où Néron les voulant exterminer, le Sénat les maintint ${ }^{56} »$. Ses connaissances juridiques, historiques et latines sont utilisées pour défendre les « financiers, traitants maltraités ${ }^{57}$ », prouver leur utilité et affirmer que le Sénat romain, modèle des parlementaires, avait en son temps défendu les financiers. Ce factum semble irrecevable pour les parlementaires, qui le 22 août donnent arrêt «qu'il seroit informé contre Catelan, Tabouret, Lefebvre et autres, et à cette fin commissaires nommés. Cet arrêt fit remuer tous les traitants, qui, s'étant assemblés, jugèrent qu'ils ne seroient jamais en repos qu'en ruinant le parlement; furent se plaindre de cet arrêt à $M$. de la Meilleraye », alors surintendant des finances, et « lui promirent fournir tout l'argent nécessaire qu'il désiroit, pourvu que l'on fit cesser le parlement ${ }^{58} »$. La banqueroute de l'État est finalement déclarée, rendant impossible le remboursement des prêts des partisans ${ }^{59}$. Le 27 août, au lendemain de la Journées des Barricades (causée par l'emprisonnement de quelques meneurs parlementaires sur ordre de Mazarin), le chancelier Séguier est pourchassé par la foule et secouru par La Meilleraye, «suivi à cheval par La Rallière, Montauron et quelques autres partisans ${ }^{60}$ ». Samuel Gaudon paraît sur le pied de guerre, au plus proche du pouvoir, au cœur de situations souvent complexes.

Le 27 janvier 1649 a lieu un événement anecdotique, mais qui va peser sur toute l'historiographie de Samuel Gaudon par sa grande diffusion littéraire à l'époque : «Le sieur de la Rallière, traitant et homme d'affaires » est « pris prisonnier en sa maison et mené à l'Hôtel de Ville et de là, dit-on, en la Bastille ${ }^{61} »$. Le témoignage de Dubuisson-Aubenay, d'une grande neutralité, fait figure d'exception. Guy Patin écrit à Charles Spon que l'on « mit hier prisonnier à la Bastille un gros et infâme partisan nommé La Rallière, lequel est rudement chargé de la haine de bien du monde, aussi était-il trop superbe et insupportable. On a pris aussi chez lui 100000 écus que l'on a portés à l'Hôtel de Ville pour aider à faire la guerre au Mazarin ${ }^{62} »$. Les journaux diffusés par les Frondeurs rendent compte de l'événement : «La Raillère insigne partisan, fort connu pour avoir esté autheur de tous les imposts sur le vin, et des taxes d'aisez; fut emprisonné à la Bastille par l'ordre de monsieur le prince de Conty ${ }^{63} »$. Le Courrier François est plus caricatural. Il y est question du

nommé la Raillere, assez conneu pour les maux qu'il a faits au peuple, tant comme principal arcboutant de Particelle, dit d'Emery, cy-devant sur intendant des finances, que pour avoir fait imposer plusieurs droits sur l'entrée du vin, et esté l'autheur, partisan, et exacteur de ce droit tyrannique imposé et levé sous le nom qu'il leur donnoit (à fausses enseignes, d'AISEZ) a esté découvert servant d'espion dans la ville pour le cardinal Mazarin ; pourquoy il a esté arresté et emprisonné à la Bastille, et encore quelques autres de pareille cabale ${ }^{64}$.

56 Ibid., p. 41-42.

57 Ibid..

58 Adolphe CHÉRUel, Mémoires de Mlle de Montpensier, petite-fille de Henri IV, collationnés sur le manuscrit autographe, avec notes biographiques et historiques, Paris : Charpentier, 1858, Journal d'Olivier d'Ormesson cité dans l'Appendice VI. Journée des barricades (1648), p. 392-397.

59 P. ClÉMENT, op. cit., 1863, p. 23.

60 Ibid., p. 392-397.

61 Journal des guerres civiles de Dubuisson-Aubenay, 1648-1652, t. I, publié par Gustave Saige, p. 133.

$62 \mathrm{BnF}$, Français 9357, «Recueil de lettres originales de Guy Patin, docteur régent en la Faculté de médecine à Paris... »; Correspondance française de Guy Patin, éditée par Loïc Capron. - Paris : Bibliothèque interuniversitaire de santé, 2015. - À Charles Spon, le 20 mars 1649.

63 Journal contenant ce qui s'est fait et passé en la cour de parlement de Paris, toutes les chambres assemblées et autres lieux, sur le sujet des affaires du temps présent es années 1648 et 1649, Paris: Gervais Alliot et Jacques Langlois, 1649, p. 151.

64 Suitte et seconde arrivée du Courier François, apportant toutes les nouvelles de ce qui s'est passé depuis sa 
Le manque d'objectivité et la surenchère dans la critique des financiers dont ont fait preuve ces auteurs compliquent leur exploitation. Ils témoignent cependant du climat de haine à l'encontre des financiers, accusés de tous les maux dont souffre le royaume. Samuel Gaudon apparaît comme un des principaux « arcboutants » du surintendant des finances Particelli d'Emery, « servant d'espion dans la ville pour le cardinal Mazarin ». Son rôle dans la perception de la taxe des Aisés a visiblement marqué les esprits et semble être à l'origine de sa notoriété, ce qui explique qu'il soit à ce point pris à partie. La déshumanisation des partisans est finalement assez impersonnelle et la seule description le concernant nommément le juge « trop superbe et insupportable ».

Deux jours après Samuel Gaudon, son associé « le sieur de Launay-Gravé fut envoyé à la Bastille par l'ordre de $\mathrm{M}$. le prince de Conti, soit en haine de ce qu'il étoit très riche et très grand partisan, ou qu'il fût soupçonné de donner des avis à Saint-Germain de ce qui se passoit dans Paris $^{65}$ ». Le pillage des demeures de partisans continue : 330000 écus sont saisis chez Étienne Pavillon le $1^{\text {er }}$ février, « comme aussi ont esté trouvez en diverses maisons de particuliers plusieurs deniers, vaisselle d'argent, meubles precieux, et autres choses que les Partisans et gens d'affaires, (qui sont fuis de Paris de peur d'estre punis) y avoient cachez ${ }^{66}$ "». L'hôtel de Samuel Gaudon a-t-il également été pillé ? Ceci expliquerait l'absence de meubles inventoriés à sa mort à la fin de l'année suivante.

Le 4 février 1649 Édouard-Jean Molé, seigneur de Champlâtreux, fait remontrer à son père Mathieu Molé, premier président du parlement de Paris, que

les sieurs de la Rallière et de Launay-Gravé étant prisonniers en la Bastille depuis plusieurs jours [...], c'étoit une inexcusable contravention à la déclaration du Roi, faite les 22 et 24 octobre 1648, par le Parlement, portant en l'article quinzième que l'on ne pourra emprisonner personne que par les formes ordinaires, c'est-à-dire, après avoir informé et décrété contre eux, et qu'étant en prison, on les interrogera devant leurs juges naturels dans les vingt-quatre heures ; que rien de tout cela n'ayant été fait pour ces deux-ci, il les faut ôter de prison ${ }^{67}$.

Alors que Molé allait à la maison de Ville faire prendre l'ordre de les libérer, « les gens de Grève avertis, allèrent à la Bastille menacer le sieur de la Rivière que si ces deux hommes sortoient, ils les mettroient en pièces à la porte, dont ils ne partiroient point. Ce qui, étant rapporté à l'Hôtel de Ville, empêcha l'expédition de l'ordre ». Leur détention doit donc se prolonger quelques jours. Peu avant le 12 février, "MM. de Beaufort, le duc de Retz, le Coadjuteur et Laigue, dînant chez Rousset, trésorier des parties casuelles, résolurent de maltraiter la Rallière ${ }^{68} »$. Hugues de Lionne, secrétaire des commandements d'Anne d'Autriche, en est informé et prévient Mazarin, qui à son tour fait prévenir Gaston d'Orléans, l'idée étant que, « s'il arrive quelque chose, l'on sache que c'était en se défendant ». L'emprisonnement de Samuel Gaudon devient une affaire d'État, dans laquelle sont impliquées les principales personnalités des deux partis opposés. Son maintien en détention témoigne de l'impuissance de l'État et de l'impunité des Frondeurs, qui tiennent la capitale.

C'est seulement près d'un mois après leur emprisonnement, le 25 février 1649, que « la Cour nomma des commissaires pour instruire le procès des nommez, la Raillere et l'Aunay-

première arrivée jusques à présent, Paris : Rolin de la Haye, 1649, p. 8.

65 Henri CourTeault, Journal de Jean Vallier, maître d'hôtel du roi (1648-1657), t. I, Paris : Renouard, Société de l'Histoire de France, 1902, p. 183.

66 Suitte et troisième arrivée du Courier François, 1649, p. 7.

67 Journal des guerres civiles de Dubuisson-Aubenay, 1648-1652, t. I, publié par Gustave Saige, p. 144-145.

68 Aimé Champollion-Figeac, Mémoires de Mathieu Molé, t. IV, Paris : Mme Ve Jules Renouard, Société de l'Histoire de France, 1857, p. 329. 
Gravé$^{69}$ ». Parallèlement on procède à la vente des meubles de Mazarin (et peut-être également de ceux de Samuel Gaudon, à moins qu'ils n'aient été pillés par la foule). D'après Célestin Moreau, « les événements marchaient cependant, et le procès ne fut pas jugé. Deux jours avant la signature des articles de la paix de Ruel, c'est-à-dire le 9 mars, les prisonniers furent relâchés ${ }^{70} »$.

Au service de Mazarin

Dès le 22 mars, Samuel Gaudon retrouve sa place au cœur des polémiques de l'époque. L'emprisonnement de Broussel, fervent Frondeur, âgé de 74 ans, passe pour avoir été décidé " sur l'advis de Catelan ou de la Raillière ». Alors que les partisans du pouvoir royal allèguent que le roi est offensé " lorsque l'on crie au meurtre sur l'oppression d'un Fuselier ou d'un Gabeleur », les Frondeurs s'offusquent que leurs adversaires qualifient « de rébellion la juste résistance que l'on fait à leurs exactions » : «Quelle parenté y a-t-il entre la Raillière et Catelan avec nos Roys ${ }^{71}$ » ? La parenté est de l'ordre de l'intérêt réciproque : tant que les troubles perdurent, les financiers ne peuvent pas percevoir correctement l'impôt, et le roi demeure privé de ses revenus.

À partir du mois d'avril, Mazarin prépare l'entrée du roi dans Paris, qui n'a lieu que le 18 août. « On craignoit que la populace, en voyant paroître le carrosse du roi, ne criât, comme elle avoit déjà fait, Vive le roi, et point de Mazarin $^{72}$ ». Le rôle de Samuel Gaudon apparaît capital puisqu'il est chargé

de distribuer aux bateliers et à d'autres gens de la lie du peuple du vin et de l'argent pour les engager à souffrir tranquillement la présence de ce ministre, qui se mit dans le carrosse du roi, sans trop savoir si les précautions qu'il avoit prises pour n'être pas insulté, auroient le succès qu'il en attendoit. Elles réussirent peut-être au-delà de ses espérances. Le roi entra dans Paris le 18 d'août 1649. Le cardinal Mazarin étoit à une des portières du carrosse avec le prince de Condé. Le peuple le regardoit avec curiosité, en disant, Voilà le Mazarin : mais aucun ne s'échappa à lui dire une injure ; au-contraire les uns disoient qu'il étoit beau et qu'ils alloient boire à sa santé ; d'autres qu'il étoit bon homme, et qu'ils avoient été trompés quand ils avoient tant crié contre lui.

L'investissement de Samuel Gaudon n'est certes pas désintéressé. Le 4 juillet 1649, Le Tellier écrit à Mazarin que « lesdits sieurs de Launay-Gravé, la Rallière et leurs associez aux entrées ont tiré des advantages très grands de Messieurs les Directeurs, lorsqu'ils ont advancé la première monstre des troupes allemandes ${ }^{73} »$. L'État doit multiplier les prêts pour financer l'armée et, pour ce, consent certains avantages aux financiers.

Le rôle politique de Samuel Gaudon est confirmé dans une lettre de Mazarin du 13 avril 1650 concernant la reddition de Bellegarde, qui était aux mains des Frondeurs. Au cours des négociations, César de Vendôme, au nom du roi, leur accorde dix jours.

Ils convinrent de donner pour ostages le comte de Coligny et un capitaine du régiment de

69 Suitte et septiesme arrivée du Courier François, apportant toutes les nouvelles de ce qui s'est passé depuis sa sixiesme arrivée jusques à présent, Paris : Rolin de la Haye, 1649, p. 5.

70 Célestin MOREAU, «Samuel Gaudon, sieur de la Raillère », Bulletin du bibliophile et du bibliothécaire, 33, Paris : Léon Techener fils, 1867, p. 278.

71 «Manuel du bon citoyen ou Bouclier de défense légitime contre les assauts de l'ennemi », 22 mars 1649, dans Célestin Moreau, Choix de mazarinades, publiés pour la Société de l'Histoire de France, t. I, Paris : Jules Renouard et $\mathrm{C}^{\text {ie }}$, 1853, p. 437-469.

72 Gabriel DANIEL, Histoire de France, t. XXII, op. cit., p. 20-21.

73 BnF, 4204, f 243 , cité dans Louis ANDRÉ, Michel Le Tellier et l'organisation de l'armée monarchique, Paris : Alcan, 1906, réédition de 1980, p. 18 . 
Monsieur le Prince, et on leur donna M. de la Rallière; à quoy M. de Vendôme apporta longtemps grande résistance, comme certainement on ne devoit pas le faire ; mais il y fut enfin porté par tous les autres officiers, qui luy representerent qu'on avoit tousjours accoustumé d'en user ainsy pour une seureté réciproque de ce dont on convenoit, et que les assiegez la donnoient plus grande, et en nombre et en la consideration des personnes.

Je sceus tout cecy à mon réveil, et n'y ayant plus de moyen d'empescher qu'on ne se fust engagé, ce que je pus faire, ce fut (aprez avoir consulté MM. les mareschaux de France qui avoient accompagné le Roy) d'ajuster les choses en sorte qu'il parut que les assiegez venoient, en suppliants, demander pardon au Roy de leur faute, laquelle Sa Majesté leur accordoit à condition de remettre la place le 21 du mois courant et de licencier, le mesme jour, toutes les troupes ; ainsy il n'est point parlé des ostages ny du pretendu secours qu'ils attendent ${ }^{74}$.

Samuel Gaudon apparaît donc comment étant de valeur légèrement inférieure « en la consideration des personnes » à un cousin éloigné de Gaspard de Coligny et à un capitaine du Grand Condé, sans pour autant que le duc de Vendôme ne juge l'échange réalisable. Il est pourtant acté, et c'est Mazarin qui trouve une autre solution pour l'éviter : preuve de l'importance de Samuel Gaudon dans la stratégie du cardinal. Mais la protection du cardinal reste soumise à certaines conditions, et le 31 juillet Claude Vanel est mis en prison à la Conciergerie « pour n'avoir satisfait à l'ordre qu'il avoit de bailler de l'argent pour la demy monstre promise à l'armée ; et La Railliere, qui est avec luy fermier des entrées de Paris, s'est absenté ou caché pour eviter prison ${ }^{75}$ ». L'inquiétude de Samuel Gaudon s'explique probablement par les difficultés qu'il rencontre à trouver des fonds pour l'État.

En effet les batailles se succèdent et le 15 décembre a lieu celle de Rethel, victoire décisive des troupes royales face aux Frondeurs alliés aux Espagnols, dont l'ampleur provoque l'union des Frondes. Avant la bataille, Pierre Gargan, intendant des finances, accompagné de Samuel Gaudon, «allantz de Rheins à Retel avec 8 hommes d'escorte seulement ${ }^{76}$ », font face à un événement imprévu raconté par deux auteurs (dont un anonyme, qui écrit de Paris, mais reste assez précis). Deux ou trois heures avant la bataille, ils tombent « imprudemment entre les mains d'un parti ennemi ${ }^{77}$ », ou sont « attaqués par les Croattes ${ }^{78}$ ». Les « croattes » sont des soldats des Habsbourg, dont ils dépendaient, mais leur nationalité est certainement imaginée d'après la violence de l'attaque, les Croates étant connus pour leur barbarie. Cinq des huit hommes d'escorte sont tués d'après l'auteur anonyme, mais Jean Vallier écrit que seul Samuel Gaudon «paya pour toute la compagnie », " ayant voulu faire le brave et tirer le coup de pistolet contre l'embuscade qui leur avoit été dressée ». Il reçoit une mousquetade ${ }^{79}$ et, « ayant fait le mort, feut laissé là ${ }^{80}$ ». Seuls Gargan et ses hommes d'escorte sont emmenés, « avec leurs chevaux et ceux de leurs carrosses, apres avoir pris au dernier 200 pistolles qu'il avoit seulement ». Le 23 décembre l'auteur anonyme écrit, toujours de Paris, que «La Railliere est encor malade à Rheins », et, le 30 décembre, qu'il « est mort de ses blessures ${ }^{81}$ ». Les blessés furent soignés à l'Hôtel-Dieu de Reims ${ }^{82}$, dont les

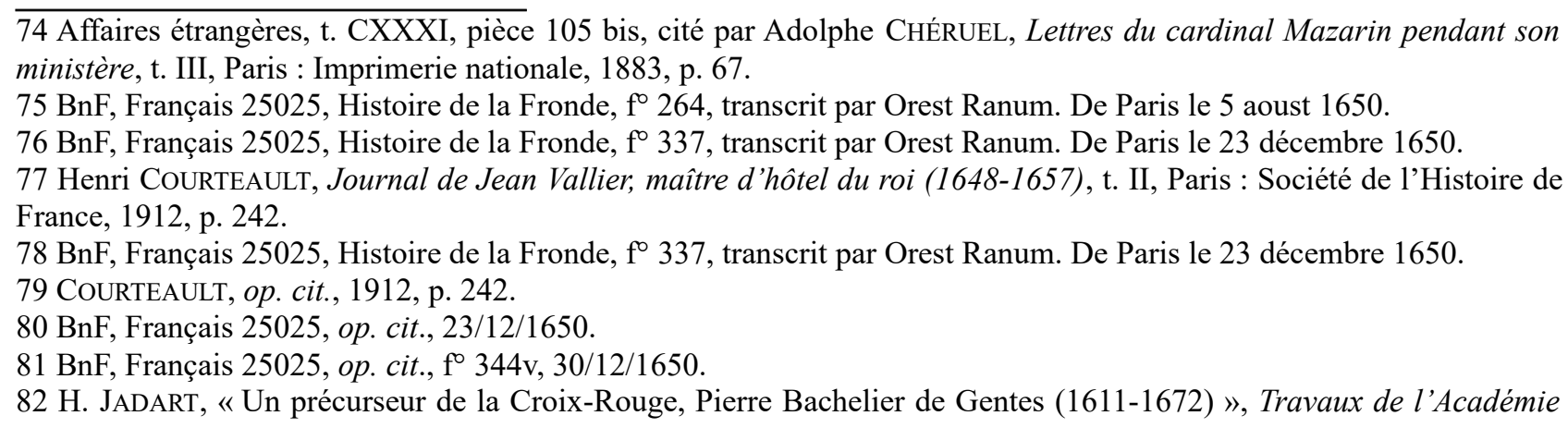


registres ne sont pas conservés. Un procès-verbal « de scellé, inventaire, description et vente de meubles en la maison de Maître La Rallière, fermier des entrées, fait par messieurs de Longueil et Berthemet » est réalisé le 29 décembre $1650^{83}$, donc sa mort était déjà connue à Paris à cette date.

Un dernier pamphlet est publié à la mort de Samuel Gaudon. Il relate le premier événement qui l'a fait connaître, à l'occasion de la naissance de Louis XIV, témoignant de son grand retentissement plus de dix ans après les faits ${ }^{84}$. Comme les autres diatribes diffusées à son encontre, il offre une vision tronquée des événements qui ne pouvait que nuire à l'étude raisonnée du personnage. Ainsi, avec le recul permis par l'analyse des archives, l'événement en question peut être perçu comme un des coups de génie de Samuel Gaudon, acte de naissance d'une carrière qui n'était plus seulement financière mais devenait de plus en plus politique :

Crocheteurs, et vous mariniers,

Porte-chaises, gaigne-deniers,

Pleurez en ce grand jour de feste,

Celuy qui devroit estre en teste

A vous faire boire à longs traicts ;

Priez les cieux qu'il soit en paix,

Pleurez vostre amy La Ralliere,

Sa cave estoit une carriere,

Vous souvient-il bien de ce jour

Que le Dauphin vint à la Cour,

Avez-vous encor la memoire

De tant de vin qu'il vous fit boire,

En vous conduisant au Bourget,

Sous le drapeau du gros Rivet.

Encore qu'il soit sous la tombe,

Vous boirez pourtant à la ronde,

Et vous soupperez en ce jour

Tous ensemble au grand carrefour.

Sa foi protestante n'étant pas même mentionnée dans les pamphlets régulièrement publiés les cinq dernières années de sa vie, quelle place a-t-elle réellement tenu pour Samuel Gaudon ? Peu d'éléments permettent d'éclairer cette question: il ne fréquente pas l'Académie de Saumur, il possède des bibles illustrées et des tableaux religieux, et il ne met jamais en avant sa foi ou le protestantisme. Pourtant, les débuts de sa carrière financière dépendent entièrement de la confiance que lui témoigne le marquis de Rochefort, et c'est dans ce milieu protestant qu'il va s'ancrer et prospérer. Les archives notariales, de même que le contrat de mariage de sa fille Madeleine, montrent qu'il fréquente aussi bien des Protestants que la noblesse parlementaire catholique. Mais c'est bien avec Georges de Clermont, d'une grande famille protestante, qu'il marie sa fille. Même treize ans après sa mort, c'est un protestant, Jean Amproux, de la petite noblesse nantaise, qu'épouse sa seconde fille Anne. La foi protestante de cette dernière est bien plus marquée que celle de sa grande sœur, qui se convertit sans difficulté au catholicisme dès la Révocation de l'Édit de Nantes, puisqu'elle devra être enfermée au couvent des nouvelles catholiques de Paris en $1686^{85}$. Le fils d'Anne et Jean Amproux, Jacques, préfère quitter la France et épouse Elisabeth Richier vers

nationale de Reims, $89^{\mathrm{e}}$ volume, Reims, 1892 , p. 376.

83 Recueil de plusieurs arrests du conseil d'Etat du roy, et autres pieces, Paris, 1702, p. 9.

84 Cartel aux bons françois pour la majorité du roy, Paris, 1651.

$85 \mathrm{AN}, \mathrm{O}^{1} 30$ 
1690 en Angleterre. Leur première fille Anne-Elisabeth est baptisée le 20 août 1696 à Londres dans l'église du Tabernacle ${ }^{86}$. Henri est baptisé le 26 septembre 1697 à Leicester Fields Chapel, dans laquelle la communauté a été déplacée ${ }^{87}$. Il deviendra ministre de l'église anglicane à Londres. Enfin, Paul Gaudon suit la trajectoire inverse de sa sœur puisqu'il se convertit très jeune au catholicisme. Il se «faict religieux ${ }^{88}$ » entre 1655 et 1658 , donc autour de ses 22 ans. À cette occasion il lègue 200000 livres à l'Hôtel-Dieu de Paris, « à la charge d'en distribuer le tiers en œuvres pies autres que l'Hostel Dieu ${ }^{89}$ ». Ces liquidités proviennent peut-être de la vente du château de la Rallière. Le testament de Madeleine en 1714, qui «donne et lègue au révérend père de la Raillière capucin » 400 livres de pension et rente viagère ${ }^{90}$, permet de confirmer qu'il est toujours vivant. Il meurt probablement peu après, étant âgé d'environ 80 ans en 1717 à la mort de sa sœur. Ce sont donc trois voies très différentes qu'ont suivi les enfants de Samuel Gaudon, mais qui ne permettent pas de mieux cerner sa foi propre. De toute évidence, son protestantisme lui a permis de bénéficier d'un réseau solide, nécessaire pour faire carrière dans la finance. Loin de tout dogmatisme, il semble avoir cultivé ce réseau sans négliger ses fréquentations catholiques, qui sont de plus en plus fréquentes à la fin de sa vie, alors qu'il est au plus proche des cercles du pouvoir.

\section{Résumé :}

Samuel Gaudon naît à Preuilly, deuxième pôle protestant de Touraine, en 1594, soit un an après le départ de Scaliger qui avait contribué à la conversion des principaux notables de la baronnie. C'est dans ce milieu protestant qu'il se forme et qu'il est repéré par le marquis de Rochefort, qui l'initie au monde de la finance. Progressivement il devient un des financiers majeurs de la Couronne, ce qui en fait une cible des parlementaires et du peuple pendant la Fronde (16481653). Sa mort brutale en 1650 intervient alors qu'il est au plus proche du pouvoir. Dès lors il sombre dans l'oubli, la seule trace qu'il laisse demeurant son château inachevé et le début de programme urbanistique l'accompagnant, au cœur de sa ville natale, juste derrière l'église abbatiale romane qu'il venait symboliquement éclipser.

\section{Abstract :}

Samuel Gaudon was born in Preuilly, the second Protestant center of Touraine, in 1594, one year after the departure of Scaliger, who had contributed to the conversion of the barony's leading figures. It was in this Protestant milieu that he was formed and later spotted by the Marquis de Rochefort, who introduced him to the world of finance. Gaudon gradually became one of the Crown's major financiers, which made him a target of the parliamentarians and the people during the Fronde (1648-1653). He died suddenly in 1650, when he was as close as he ever would be to the reigning power. Since then he has fallen into oblivion, the only remaining trace of his life being an unfinished castle and the start of an accompagnying urban planning program in the heart of his native town, just behind the Romanesque abbey church that he had symbolically eclipsed.

\footnotetext{
86 Tulot, Jean-Luc, Une communauté protestante rurale de Bretagne à la veille de la Révocation de l'Edit de Nantes : Blain, Nantes, Cercle généalogique de l'Ouest, 1991.

87 Smiles, Samuel, The Huguenots - Their Settlements, Churches and Industries in England and Ireland, 2013 (réédition).

88 AN, E 389A.

89 Möring, Michel, Collection de documents pour servir à l'histoire des hôpitaux de Paris, Tome premier, Délibérations de l'ancien bureau de l'Hôtel-Dieu, Paris, 1881, p. 133.

90 AN, M.C., Étude LXXVII, 150.
} 